\title{
Utilización de Wolfram Mathematica la resolución de problemas de Ingeniería
}

\author{
Samuel Sánchez Caballero ${ }^{1}$, Miguel Ángel Sellés Cantó ${ }^{1}$, Rafael Pla \\ Ferrando $^{1}$ y Sergi Montava Jordà ${ }^{1}$ \\ ${ }^{1}$ Escuela Politécnica Superior de Alcoy. Universitat Politècnica de València
}

\begin{abstract}
Machine Design teaching has been traditionally related with the use of lots of calculation formulae, graphs and tables whose manipulation involves a human error. The use of Mathematica in teaching allows the students to replace this mathematical apparatus with a few code lines. This lets the professor to be focused in the main design concepts, allowing him to assess the student competence instead if its calculator or reading skills. As a result of this experience, not only the students grades have been improved, but also the exams have become a way to discriminate the students that have reached the problem-solving competence.
\end{abstract}

\section{Resumen}

La enseñanza del Diseño de Máquinas ha estado tradicionalmente ligada a la utilización de numerosas fórmulas de cálculo, gráficas y tablas cuya manipulación conlleva un error humano inherente. La introducción de Mathematica en la docencia permite a los estudiantes reemplazar todo este aparato matemático, con unas pocas líneas de código. Esto posibilita que el profesor se centre en las cuestiones fundamentales del diseño, permitiéndole evaluar realmente la competencia del alumno a la hora de resolver problemas y no su habilidad para teclear una calculadora o leer en gráficas y tablas. Como resultado de esta experiencia, no solo se ha logrado una mejora en los resultados de los alumnos, sino que los exámenes se han convertido realmente en un sistema que discrimina a los alumnos que han adquirido la competencia de resolución de problemas de los que no.

Keywords: Mathematica, Diseño de Máquinas, Resolución de Problemas, Ingeniería. 


\section{Introducción}

El presente trabajo se desarrolla a partir de la experiencia adquirida en la docencia de la asignatura de Teoría y Diseño de Máquinas (Diseño de Máquinas en los planes antiguos) durante los últimos dieciocho años. Dicha asignatura pertenece al grado de Ingeniería Mecánica y es obligatoria y anual, con un peso total de 9 créditos, estando repartidos al $50 \%$ entre las clases de teoría y prácticas. La asignatura es a su vez punto de control de la competencia de Resolución de problemas"Resolución de problemas" del citado grado, por lo tanto se trata de una asignatura donde los alumnos hacen de un uso intensivo de la calculadora en los exámenes.

La experiencia adquirida durante los primeros doce años de docencia de la asignatura mostraba que los resultados obtenidos por los alumnos en el apartado de problemas eran especialmente malos, pese a haberse planteado diferentes estrategias de mejora. Dichas estrategias de mejora estuvieron orientadas fundamentalmente a la ampliación del abanico de problemas resueltos disponible, y al planteamiento de problemas con resolución autónoma por parte del alumno. Pese a estas acciones, la tasa de abandono se mantenía especialmente elevada (por encima del $40 \%$ ), a la vez que la nota media se mantenía por debajo del tres, a lo largo de los años.

Partiendo de esta situación, este trabajo analiza inicialmente las posibles causas de estos malos resultados para plantear como innovación docente la introducción del software Wolfram Mathematica en la resolución del apartado de problemas de la asignatura. Se analizan diferentes etapas seguidas en la introducción del software, desde la más elemental donde se reproducía el esquema de resolución manual, hasta la más avanzada con el empleo de paquetes y funciones recursivas. Como resultado de este trabajo se ha logrado una reducción significativa de la tasa de abandono, así como una mejora creciente en la nota media obtenida por los alumnos.

\section{Objetivos}

El objetivo principal de este trabajo es la reducción de la elevada tasa de abandono, así como la mejora de los resultados obtenidos en el apartado de problemas de la asignatura de Teoría y Diseño de Máquinas.

\section{Desarrollo de la innovación}

Análisis de las causas

La ausencia de mejoras en los resultados provocó que a finales del curso 2012/13 se decidiera hacer un análisis de la problemática asociada a la asignatura. La Figura 1 muestra los resultados obtenidos entre los cursos 2009 y 2012. Como puede apreciarse, las estrategias de mejora seguidas durante estos años elevaron la nota media de los alumnos aprobados. Sin embargo, la tasa de alumnos que no superaban el examen de problemas era muy significativa, siendo especialmente remarcable la tasa de alumnos que o bien obtenían menos de un punto, o bien ni siquiera se presentaban al examen. Esta tasa se situaba entre el 40 y el $50 \%$, sin que se se apreciase ningún efecto de las estrategias aplicadas sobre la misma. En base a estos resultados se decidió realizar un análisis de las posibles causas, determinando entre ellas las siguientes: 

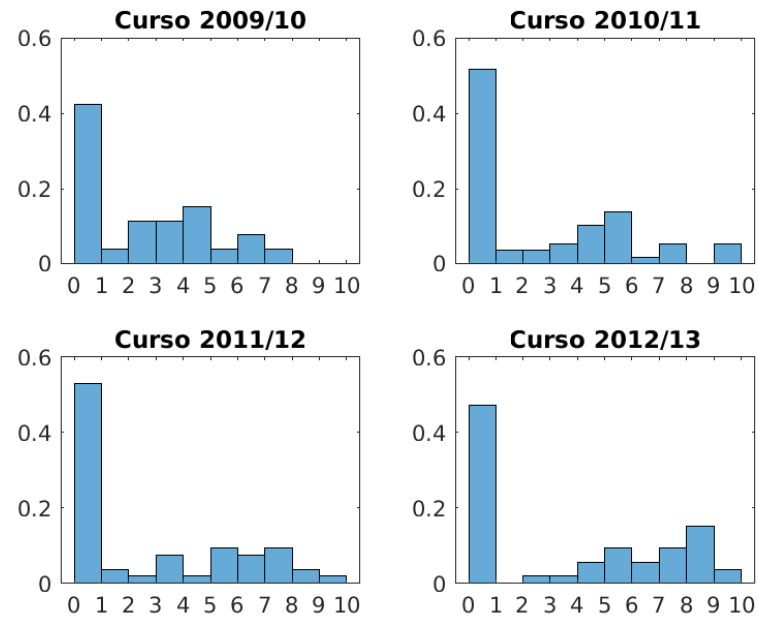

Fig. 1: Distribución de notas entre los cursos 2009 y 2012

1. La cantidad de formulación que deben manejar es muy elevada por lo que existe una probabilidad bastante grande de que los alumnos cometan errores en la transcripción de las fórmulas.

2. la resolución de problemas requiere de múltiples cálculos concatenados y dependientes entre sí, de forma que un resultado erróneo provoca un error en cascada en la solucion.

3. La resolución de problemas a mano requiere de mucho tiempo y hay una gran posibilidad de equivocarse. Si, por ejemplo, nuestra tasa de error en un cálculo simple es del $1 \%$, cuando encadenamos 20 cálculos, la probabilidad de error se incrementa al 18,2\%. De este modo resulta bastante difícil obtener la máxima puntuación.

4. Existen otras fuentes adicionales de error, como el manejo de tablas o gráficos.

5. La resolución de determinados apartados como la obtención de tensiones principales, el calculo de holgura en cojinetes o la distancia entre centros de engranajes, requiere de la utilización de métodos numéricos de resolución de ecuaciones ya que no tienen solución algebraica.

6. En el mundo laboral, los cálculos se raramente se realizan a mano.

7. De algún modo, estamos evaluando su habilidad para manejar la calculadora, no su competencia para resolver problemas. 
Estrategias de mejora. Primera etapa: 2013-2014

A partir de estas observaciones, así como de la experiencia obtenida durante una estancia en el verano del curso 2011/12 en la Universidad de Notre Dame (IN, USA) donde los alumnos utilizaban el software Wolfram Mathematica como herramienta de resolución de problemas (Cheah y col. 2016; Vetyukov 2016; Vinogradov 2000; Rus, Veresiu y Mereuta 2014), se decidió empezar a emplear este software como herramienta de apoyo en la resolución de problemas de la asignatura. Con objeto de poder comparar los resultados obtenidos, el sistema de evaluación, el tipo de examen y el peso de los diferentes apartados se ha mantenido estable a lo largo de los cursos, antes y después de la incorporación de la herramienta en la docencia. Sin embargo, la forma de utilizar el software dentro de la asignatura ha variado durante los diferentes cursos. Seguidamente se describen las acciones seguidas en cada una de las etapas. En una primera etapa, nos centramos en los apartados de mayor complejidad como el cálculo de tensiones principales, diferentes factores de fatiga, el cálculo generalizado de diferentes elementos de máquinas. A modo de ejemplo, se muestra el desarrollo del cálculo de tensiones principales empleando Wolfram Mathematica.

El cálculo de tensiones principales es uno de los primeros cálculos requeridos en la resolución de problemas y requiere de la diagonalización del tensor de tensiones $\mathbf{T}$ :

$$
\boldsymbol{\sigma}=\left[\begin{array}{ccc}
\sigma_{x} & \tau_{x y} & \tau_{x z} \\
\tau_{x y} & \sigma_{y} & \tau_{y z} \\
\tau_{x z} & \tau_{y z} & \sigma_{z}
\end{array}\right] \cdot\left[\begin{array}{c}
\mathbf{i} \\
\mathbf{j} \\
\mathbf{k}
\end{array}\right]=\mathbf{T} \cdot \mathbf{u}
$$

De donde se obtiene la ecuación característica:

$$
\sigma^{3}-I_{1} \sigma^{2}+I_{2} \sigma-I_{3}=0
$$

Cuyos coeficientes son:

$$
\begin{aligned}
& I_{1}=\sigma_{x}+\sigma_{y}+\sigma_{z} \\
& I_{2}=\sigma_{x} \sigma_{y}+\sigma_{x} \sigma_{z}+\sigma_{y} \sigma_{z}-\left(\tau_{x y}^{2}+\tau_{x z}^{2}+\tau_{y z}^{2}\right) \\
& I_{3}=\|T\|
\end{aligned}
$$

La resolución de esta ecuación característica requiere de utilizar métodos numéricos, o bien una compleja solución algebraica derivada del cambio de sistema de referencia al espacio de Haigh-Westergaard.

Por contra, el cálculo con Mathematica es mucho más simple y solo requiere de una sencilla instrucción:

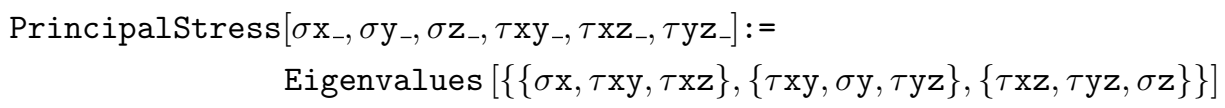

De este modo, el programa se empleaba a modo de herramienta que permitía calcular los apartados más complejos del problema. 
Estrategias de mejora. Segunda etapa: 2014-2015

Durante esta etapa se desarrolla la formulación completa de la asignatura, a excepción de los factores que aparecían en tablas o gráficas. Los ejercicios se desarrollaban en la pizarra mediante el método tradicional para ser posteriormente resueltos siguiendo los mismos pasos que en la resolución manual y comparando los resultados. Durante esta etapa se inician dos sesiones de prácticas introductorias al programa dadas las carencias de formación detectadas en la etapa anterior. También se introduce el desarrollo de talleres virtuales y representaciones tridimensionales que han resultado muy útiles para ilustración de los diferentes conceptos teóricos. A continuación se muestran varios de ellos.

1. Teorias de fallo bajo estado tensional plano para materiales frágiles: el que el alumno puede determinar, para cualquier estado tensional plano, si las tensiones principales asociadas generarán la rotura del material gráfica y numéricamente. También proporciona los valores de las tensiones equivalentes y los coeficientes de seguridad.

http://labmathematica.upvnet.upv.es/eslabon/Ejercicio.asp?do=Teorias_fallo_fragil

2. Teorias de fallo bajo estado tensional plano para materiales dúctiles: el alumno puede determinar, para cualquier estado tensional plano, si las tensiones principales asociadas generarán la fluencia del material gráfica y numéricamente. También proporciona los valores de las tensiones equivalentes y los coeficientes de seguridad.

http://labmathematica.upvnet.upv.es/eslabon/Ejercicio.asp?do=Teorias_fallo_ductil

3. Determinación de la tensión alternante pura equivalente a un estado tensional compuesto: el alumno puede determinar, en función de las componentes medias y alternante de tensión, la tensión equivalente pura gráfica y numéricamente. http://labmathematica.upvnet.upv.es/eslabon/Ejercicio.asp?do=Tension_alternate_pura

4. Teorías de fallo por fatiga: el alumno puede determinar, en función de las componentes medias y alternante de tensión, si estas generarán la rotura del material gráfica y numéricamente.

http://labmathematica.upvnet.upv.es/eslabon/Ejercicio.asp?do=Teorias_fallo_fatiga

Estrategias de mejora. Tercera etapa: 2015-2016

En base a los buenos resultados obtenidos en las etapas anteriores se continúa profundizando en la herramienta y se introduce el empleo de funciones recursivas. Como se ha indicado anteriormente, el cálculo requiere en muchos casos del cálculo de diversos factores intermedios para obtener el factor final, el cual es el realmente interesante. El cálculo de factores intermedios requiere de la ejecución de instrucciones intermedias que requieren de tiempo y pueden inducir a cierto error en caso de olvidar el cálculo de alguno de estos factores. A modo de ejemplo se muestra uno de estos cálculos.

El cálculo del concentrador de tensiones corregido a fatiga requiere el cálculo previo de las constantes de Neuber y la sensibilidad a la entalla, las cuales se calculan tradicionalmente de la siguiente forma:

La constante de Neuber (Neuber 2001) se calcula mediante la fórmula: 


$$
\sqrt{a}=\left\{\begin{array}{cl}
-0,32865+34,5452 S_{u t}^{-0,60977} & \text { para el acero } \\
-0,29486+77,4708 S_{u t}^{-0,78374} & \text { para el aluminio recocido } \\
0,0634+101,97946 S_{u t}^{-0,81409} & \text { para el aluminio endurecido }
\end{array}\right.
$$

La sensibilidad a la entalla se calcula en función de la constante de Neuber mediante la ecuación:

$$
q=\frac{1}{1+\frac{\sqrt{a}}{\sqrt{r}}}
$$

Finalmente, y a partir del valor de $K_{t}$, obtenido mediante gráficas (Peterson 1966): se el valor del concentrador de tensiones corregido a fatiga $K_{f}$ mediante la expresión:

$$
K_{f}=1+q\left(K_{t}-1\right)
$$

El desarrollo de las siguientes funciones recursivas, permite el cálculo del factor $K_{f}$ mediante una única llamada a la función (la última) y sin tener que calcular los valores intermedios de la constante de Neuber y la sensibilidad a la entalla:

$$
\begin{aligned}
& \text { Fneubercons [material_, Sut_] : =Which[acero == material, } \\
& -0,32865+34,5452 \mathrm{Sut}^{\wedge}-0,60977 \text {, aluminio }==\text { material, } \\
& -0,29486+77,4708 \mathrm{Sut}^{\wedge}-0,78374 \text {, duraluminio }==\text { material, } \\
& \left.0,0634+101,97946 \text { Sut }^{\wedge}-0,81409\right] \\
& \text { Fq[material } \left., \text { Sut }_{-}, \mathbf{r}_{-}\right]:=1 /(1+\text { Fneubercons[material, Sut] } / \text { Sqrt }[\mathrm{r}]) \\
& \text { FKf } \left.\left[\text { material_,Kt_,Sut,_, } \text { Kt }_{-}\right]:=1+\text { Fq[material,Sut,r }\right](\text { Kt-1) }
\end{aligned}
$$

Estrategias de mejora. Cuarta etapa: 2016-2017

Mientras que en la etapa anterior se siguió utilizando el esquema de resolución de problemas tradicional, pese a la introducción de algunas funciones recursivas, en esta etapa se extiende el uso de funciones recursivas a la resolución de problemas. La introducción de esta metodología de cálculo supone un cambio de paradigma ya que la resolución de problemas deja de seguir los pasos tradicionales, requiriendo un nivel de abstracción mayor por parte de los alumnos. Durante esta etapa se inicia también la conversión a forma de función de las diferentes tablas y gráficas empleadas, ya que los resultados de la etapa anterior muestra que la mayoría de los errores cometidos por parte de los alumnos se centra en el manejo de tablas o la lectura de gráficas. 
Estrategias de mejora. Quinta etapa: 2017-2018

Durante el curso actual se ha introducido el empleo de aplicaciones en Wolfram Mathematica desarrollados expresamente para la asignatura. Las aplicaciones de Mathematica constan por una parte de todo el conjunto de funciones necesarias para realizar los diferentes cálculos, así como de un sistema de ayuda donde se describen las diferentes funciones, sus parámetros y limitaciones de uso. La Figura 2 muestra un ejemplo de la aplicación para el cálculo de concentradores de tensiones.

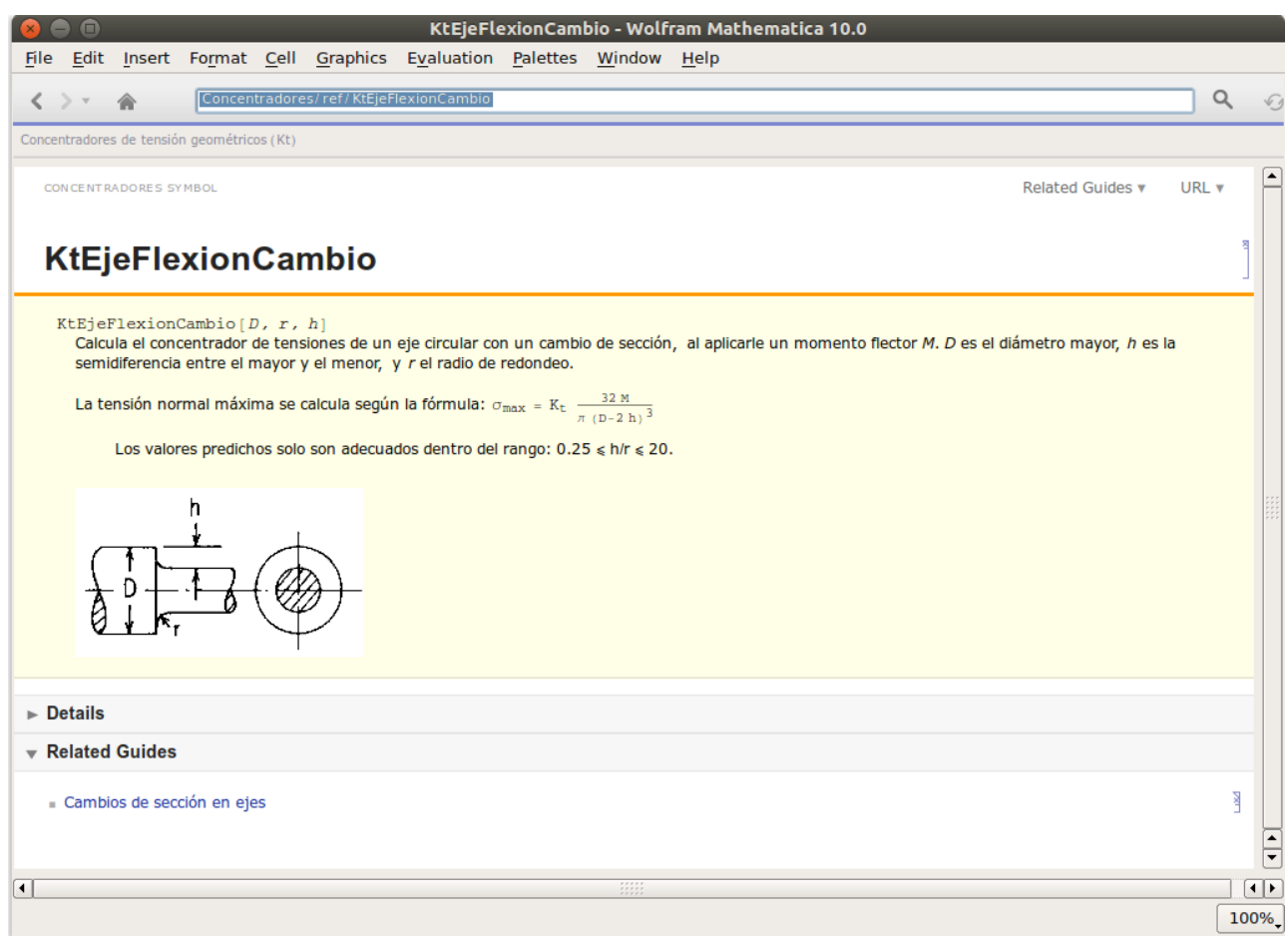

Fig. 2: Ejemplo de la ayuda de la aplicación para el cálculo de concentradores de tensiones

\section{Resultados}

La Figura 3 muestran la distribución de notas la asignatura durante los últimos cuatro cursos. Como puede apreciarse, el porcentaje de alumnos con una nota inferior a uno o que no se presentan disminuye significativamente desde el primer curso, pasando del $40 \%$ existente antes de la introducción del programa, al $10 \%$ en los dos últimos cursos. Así mismo, puede apreciarse como los alumnos que aprueban la asignatura, obtienen notas significativamente más altas.

Por otro lado, la Figura 4 y la Tabla 1 muestran la evolución de la nota media durante los últimos ocho años. Como puede observarse, la nota media de la asignatura se incrementa año tras año, pasando de 2,44 a 6,72 puntos durante el último curso, siendo 
especialmente significativo el incremento de la misma a partir del curso 2013/14, coincidiendo con la introducción del software en la asignatura.
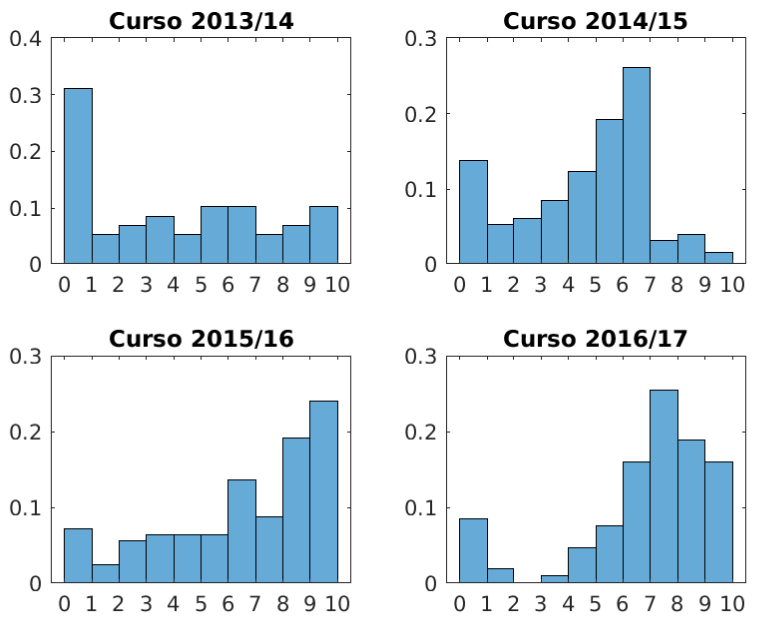

Fig. 3: Distribución de notas entre los cursos 2013 y 2016

Tabla 1: Evolución de la media y la desviación típica

\begin{tabular}{lcccccccc} 
Curso & $09 / 10$ & $10 / 11$ & $11 / 12$ & $12 / 13$ & $13 / 14$ & $14 / 15$ & $15 / 16$ & $16 / 17$ \\
\hline Media & 2,44 & 2,50 & 2,54 & 3,49 & 4,00 & 4,50 & 6,41 & 6,72 \\
Desv. Típica & 2,47 & 2,98 & 3,13 & 3,58 & 3,41 & 2,46 & 2,92 & 2,59 \\
\hline
\end{tabular}

Finalmente, los resultados de la implantación de los aplicaciones y paquetes durante este curso no se encuentran actualmente disponibles, aunque se prevee sean mejores debido a que se reduce la posibilidad de cometer errores al eliminar la lectura de gráficas o la interpolación en tablas.

\section{Conclusiones}

Después de cuatro cursos utilizando Wolfram Mathematica como herramienta de resolución de problemas, el resultado ha sido mas que satisfactorio, no solo porque se ha conseguido mejorar los resultados de la asignatura, sino porque los mismos alumnos proporcionan un feed-back positivo al respecto. La gran mayoría siguen utilizando el programa en otras asignaturas o en el desarrollo de sus Trabajos de Fin de Grado. Así mismo, en vista de los resultados obtenidos, otros profesores del grado se han implicado en la utilización del programa en sus asignaturas. Finalmente, la experiencia se ha hecho extensiva al resto de profesores de la escuela, programándose varios cursos de formación sobre el programa con más de veinticinco profesores implicados.

Evidentemente, la experiencia también ha presentado varios aspectos negativos que deben también ser remarcados. Uno de ellos es que los alumnos que no dominan la asignatura, tampoco lo hacen con el programa, lo cual les supone una piedra de tropiezo, obteniendo resultados incluso inferiores. Normalmente estos alumnos tienen 


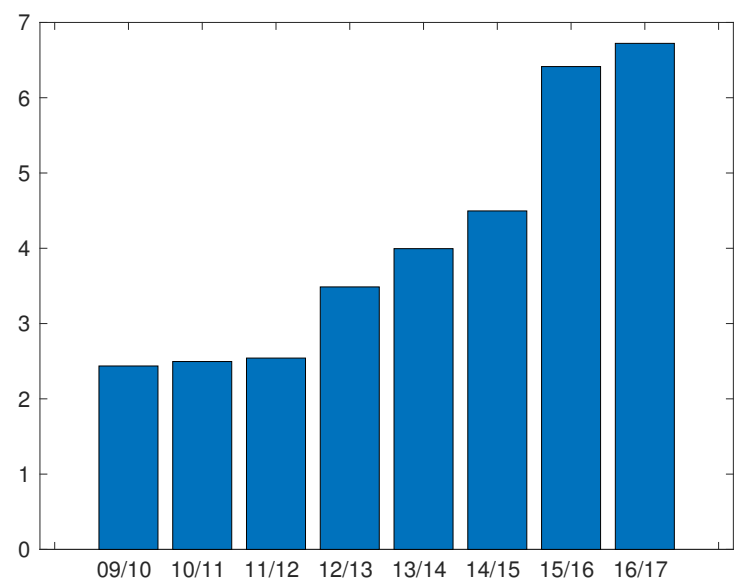

Fig. 4: Evolución de la nota media desde el curso 2009/10 al 2016/17

la falsa impresión de que el programa resuelve los problemas por si mismo. Finalmente, una pequeña proporción estudiantes intentan copiar. Evidentemente, cuando se utiliza un soporte electrónico esta opción parece más tentadora, por lo cual requiere de un esfuerzo extra por parte del profesor para evitarlo. A este respecto, el propio programa incorpora un historial donde se registra temporalmente todos y cada uno de los cambios realizados en la hoja, lo cual puede servir para revisar estos casos cuando existan dudas.

\section{Agradecimientos}

Los autores desean agradecer al equipo de Wolfran Mathematica Europa y en especial a Maria Dimitrova su labor de soporte y colaboración con el programa.

\section{Referencias bibliográficas}

Cheah, C. T. y col. (2016). "Selection of software for mechanical engineering undergraduates". En: AIP Conference Proceedings 1754.1, pág. 060002. DOI: 10.1063/1. 4958443. eprint: https://aip.scitation.org/doi/pdf/10.1063/1.4958443.

Neuber, Heinz. (2001). Kerbspannungslehre : Theorie der Spannungskonzentration ; genaue Berechnung der Festigkeit. Berlin: Springer.

Peterson, Rudolph Earl (1966). Stress concentration design factors; charts and relations useful in making strength calculations for machine parts and structural elements. New York: Wiley. 
Rus, M., S. Veresiu y E. Mereuta (2014). "Using mathematica program in teaching mechanisms". En: vol. 17. cited By 0, págs. 531-540. DOI: 10.1007/978-3-31901845-4_53.

Vetyukov, Yury. (2016). Nonlinear Mechanics Of Thin-Walled Structures : asymptotics, direct approach and numerical analysis. Springer.

Vinogradov, Oleg. (2000). Fundamentals of kinematics and dynamics of machines and mechanisms. Boca Raton; London: CRC Press. 